\title{
AUTOMATIC EBSP ANALYSIS FOR RECRYSTALLIZATION STUDIES
}

\author{
D. JUUL JENSEN \\ Materials Department, Ris $\varnothing$ National Laboratory, DK-4000 Roskilde Denmark
}

\begin{abstract}
The potential of local texture techniques for investigations of recrystallization are reviewed. The need for automatization of such techniques is discussed. It is shown how orientation measurements using one of these techniques, the electron back scattering pattern (EBSP) technique, can be made fully automatic.
\end{abstract}

KEY WORDS Recrystallization, image processing, EBSP.

\section{INTRODUCTION}

Traditionally, recrystallization has been studied using mostly microscopy and texture techniques. It has, however, proved to be very complicated to relate the microstructure development to the bulk texture results. Local texture techniques, which give information about the crystallographic orientation within selected areas of the microstructure can be used to link the microstructure to the bulk texture results and may thereby help to further the understanding of the recrystallization mechanisms.

\section{LOCAL TEXTURE TECHNIQUES AND RECRYSTALLIZATION}

\subsection{Techniques}

Several techniques may be used in the determination of the crystallographic orientation in selected local areas of the microstructure. Most of these techniques are electron microscopy techniques by which the microstructure is seen directly in the microscope and the orientation of individual subgrains/grains is determined from analysis of electron diffraction patterns. An overview of such techniques is given in Table 1.

The TEM techniques (Heimendahl, Bell and Thomas, 1964, Hirsch, Howie, Nicholson, Pashley and Whelan, 1965, Chapman and Stobbs, 1969, Ralph and Ecob, 1984, Humphreys, 1988) are characterized as having a very good spatial resolution, i.e. the crystallographic orientation of even small subgrains in highly deformed structures may be determined. TEM investigations are often limited by difficulties in preparing large areas of electron transparent material. However, new thinning techniques (Klepeis, Benedict and Anderson, 1988), as applied to semiconductor materials, may also give improved TEM foils in metals.

With the SEM techniques (Newbury and Yakowitz, 1975, Hall and Hutchinson, 1980, Dingley, 1981, Humphreys, 1988, Juul Jensen and Randle, 1989) large 
Table 1 The main characteristics of three classes of local texture techniques

\begin{tabular}{|c|c|c|c|c|}
\hline & & \multirow[t]{2}{*}{$\begin{array}{l}\text { Spatial } \\
\text { resolution }\end{array}$} & \multicolumn{2}{|c|}{$\begin{array}{l}\text { Angular } \\
\text { accuracy }\end{array}$} \\
\hline & & & Spot & Kikuchi \\
\hline TEM & $\begin{array}{l}\text { selected area diffraction } \\
\text { micro diffraction } \\
\text { convergent beam }\end{array}$ & $\begin{array}{l}2 \mu \mathrm{m} \\
20 \mathrm{~nm} \\
2 \mathrm{~nm}\end{array}$ & $\begin{array}{l}2^{\circ} \\
0.2^{\circ} \\
-\end{array}$ & $\begin{array}{l}0.1^{\circ} \\
0.1^{\circ} \\
0.1^{\circ}\end{array}$ \\
\hline TED & $\begin{array}{l}\text { transmission electron } \\
\text { diffraction (pole figures) }\end{array}$ & $\geq 5 \mu \mathrm{m}$ & $2^{\circ}$ & \\
\hline SEM & $\begin{array}{l}\text { channeling } \\
\text { electron back scattering (EBSP) }\end{array}$ & $\begin{array}{r}10 \mu \mathrm{m} \\
1 \mu \mathrm{m}\end{array}$ & $\begin{array}{r}0.5^{\circ} \\
1^{\circ}\end{array}$ & \\
\hline
\end{tabular}

sample areas can be inspected routinely. However, the orientation of subgrains smaller than $\sim 1 \mu \mathrm{m}$ can not be determined, and fine details of the microstructure are not as well resolved as in the TEM.

The geometry of an X-ray transmission texture camera may be applied for transmission electron diffraction and a pole figure for a selected region $(5 \mu \mathrm{m})$ can be obtained (Humphreys, 1983, Weiland and Schwarzer, 1984). The principle of this technique is to measure the diffracted intensity along an ( $h k l)$ Debye Scherrer ring, and to scan the tilting of the thin foil specimen from $0-60^{\circ}$ to cover as large a part of the $\{h k l\}$ pole figure as possible. Typically, the texture within $5-20 \mu \mathrm{m}$ selected local regions of the microstructure is determined in this way (Humphreys, 1988). Once the pole figure measurement is completed, it is possible to identify elements in the microstructure corresponding to specific parts of the pole figure (Weiland, 1991a). This technique, in the following referred to as "TED", is ideal for measurements of all orientations within a selected region of the microstructure.

Other techniques, like the Kossel technique (Peters and Ogilvie, 1965, Bellier and Doherty, 1977, Dadson and Doherty, 1992) and synchrotron radiation techniques (Gottstein, 1988), have also been used to measure local crystallographic orientations. However, as no simultaneous information about the microstructure is obtained with these techniques, they will not be considered here.

\subsection{Recrystallization}

The three types of local texture techniques discussed above, the TEM, SEM and TED techniques, can each be used to address important aspects of the recrystallization process. In Table 2 the present potential of TEM/Kikuchi, TED and SEM/EBSP for the study of the various aspects of recrystallization are suggested. These aspects-nucleation, growth and recrystallized structure-will be discussed in the following.

Nucleation: Important questions which may be answered from the acquisition of local texture information are: i) What sites in the deformed microstructure are potential nucleation sites? ii) Is there a relationship between the nucleation site and the orientation of the nuclei? iii) What are the orientation relationships between a nuclei and the surrounding matrix? 
Table 2 Potentials of 3 local texture techniques for studies of recrystallization

\begin{tabular}{llll}
\hline & $\begin{array}{l}\text { TEM } \\
(\text { Kikuchi })\end{array}$ & TED & $\begin{array}{l}\text { SEM } \\
(\text { EBSP })\end{array}$ \\
\hline Nucleation & + & + & + \\
Growth & - & - & + \\
Recrystallized structure & - & - & + \\
\hline
\end{tabular}

It is very well known that two criteria must be fulfilled for a site to be a potential nucleation site (question i) (above); a large lattice misorientation is needed, and the driving force must be sufficient for the nucleus to grow. Also it is fully established that original grain boundaries, large second phase particles, transition bands and shear bands are nucleation sites (e.g. Doherty, 1980). However, it is not understood why for example only some of these sites become operational (e.g. only few nuclei develop along a grain boundary), why the nuclei may develop near but not at the transition band (Driver, 1992), or why some nuclei for no obvious reason stop growing very early in the recrystallization process (Bay and Hansen, 1979, Juul Jensen, Hansen and Humphreys, 1985, Humphreys and Juul Jensen, 1986). For studying this, it is of interest to measure the orientations of the individual deformation subgrains near the potential nucleation sites. For materials deformed to medium and high strain only the TEM techniques have the required spatial resolution, whereas for materials deformed to a low strain or by hot deformation, SEM/EBSP becomes important. Furthermore, TED and SEM/EBSP may be used for a spatially more coarse mapping of orientation distributions in the deformed structure, which may be used in the explanation of the limited growth of some nuclei.

All three types of techniques may also be used in studying question ii) and iii) above. The TEM techniques can give the most precise and detailed results to both questions. However, at present a serious draw back is the limited sample area in a typical thin foil and therefore the limited chance of finding nuclei. Furthermore, for studies related to question ii), the time involved in measuring all the individual orientations of nuclei and surrounding subgrains in the matrix may be overwhelming. For this the TED technique is clearly much more effective. With SEM it is straightforward to find, and to determine, the orientation of the nuclei. Due to recovery in the matrix during annealing to the start of nucleation, orientations of matrix subgrains can in general also be measured by EBSP (Hjelen, Ørsund and Nes, 1991). However, the microstructure is not so clearly resolved as in the TEM. For example it may be difficult to see whether a nucleus has developed at a transition band or if it is at an intragranular segment of a high angle grain boundary or a microband. As such it may be difficult to answer questions i) only using SEM. For a few particularly interesting nuclei, it may be valuable to locate the position of the nucleus in the SEM, and then produce a TEM foil from that region (Lindbo, 1991) and continue the investigation by TEM.

Growth. Important microstructure/texture questions are: i) How does the growth rate depend on the annealing time and does the growth rate and time dependence vary with the orientation of the grain? ii) Are there preferred orientation relationships between a growing grain and the matrix? 
For studying the question i) the SEM/EBSP technique is very well suited since the size and orientation of the grains is easily determined. From a series of partly recrystallized specimens, the average growth rate $G$ of each group of grain orientations may be determined using the equation (English and Backofen, 1964, DeHoff, 1968):

$$
\frac{d X}{d t}=G \cdot A
$$

where $X$ is the volume fraction recrystallized, $t$ is the time and $A$ is the interfacial area separating recrystallized from unrecrystallized grains (the migrating boundary area).

Using this method, it is straight forward, but very time consuming, to determine statistically reliable results for the growth rates.

Question ii) is of course very difficult to answer. The local texture techniques considered here cannot be used for in-situ experiments and conclusions can therefore only be reached very indirectly.

Recrystallized structure local texture techniques may be used to study the following important questions i) Is there a relationship between the grain size and grain orientation? ii) What is the grain boundary geometry?

Also for these types of investigations the SEM/EBSP technique seems to be the most powerful (see Table 2), since the other techniques are restricted by the limited sample area.

The size and orientation of the grains may be determined by first measuring the size of a grain looking directly at the microstructure. Then the grain orientation may be determined from the corresponding EBSP pattern. This procedure requires a series of microscope manipulations and is therefore rather time consuming. A faster procedure is to stay in "EBSP-mode" and make line-scans by either moving the beam or the sample. When the pattern changes a new grain is reached. In this way the orientations are determined directly and linear intercept values for the grain sizes are obtained.

The potentials of SEM/EBSP for characterization of the grain boundary geometry are discussed elsewhere in these proceedings (Randle, 1992, Watanabe, 1992) and will not be considered here.

\section{AUTOMATED TECHNIQUES AND EBSP}

\subsection{Automated Techniques}

Reasonably fast and user-friendly procedures for semiautomatic analysis of the orientation patterns (Kikuchi, EBSPs etc.) are available for the local texture techniques discussed above (Venables, Harland and bin-Jaya, 1976, Carr, 1982, Schwarzer and Weiland, 1984, Dingley, Longden, Weinbren and Alderman, 1987, Schmidt, Bilde-Sørensen and Juul Jensen, 1991, Weiland 1991b). An important question is therefore, if it is worthwhile to develop fully automatic indexing procedures?

The answer to the question probably depends on the type of investigation in mind. For the recrystallization studies discussed above, the answer may be an equivocal yes. For example, for the TEM studies of the deformation microstruc- 
ture and the SEM studies of nucleation, it is very important to locate precisely in the microstructure where for example the subgrain or nucleus is. A "blind" automatic scan through the structure would in general only give little relevant information. In these cases, it would be convenient to have an image processing procedure which directly could index a given pattern without the need for the operator to mark bands or poles. However, the extra time and effort required in marking the patterns using the semiautomatic procedures are in general of minor importance. For the SEM investigations of the growth rates and size-orientation relationships, on the other hand, line scans through the structure (in the EBSP mode) give the required information-the orientations, the sizes (linear intercept values) and the migrating boundary areas A (Eq. (1)) of the grains. It is of course possible to do such scans semiautomatically, but to collect statistically reliable data requires several days or often weeks of very exhausing work. Automatisation of the indexing of EBSP is thus clearly necessary. Such automation would not mean that a sample is placed in the SEM and the results come out directly. It is believed that for the investigations discussed, an initial microstructural inspection is always necessary. By this inspection, it is determined along which line or lines the scan should be made and what the appropriate step size is. The aim of the automation is then to develop a system, which, after calibration, allows automatic scanning along the predetermined line and determination of all orientations without any involvment of a human operator.

\subsection{Experimental Set-up}

The principles and experimental set-up for the EBSP technique are very well known (Dingley, 1981, 1984), and shall not be described here. However, it should be noted that the quality of the EBSPs depends strongly on the position in which the phospor imaging screen is placed in the SEM (Alam, Blackman and Pashley, 1954, Hjelen, 1990). This is illustrated in Figure 1. Here EBSPs taken with the phosphor screen mounted in the rear port (a standard commercial version of the EBSP set-up) and in the side port are shown. The difference in quality of the two patterns relates to the lower position of the screen relative to the sample when the side port is used (Figure 1b). Here the intensity of back-scattered electrons is higher, and the signal to noise ratio of the EBSP is therefore better. All following EBSP to be shown in this paper is taken with the phospor screen mounted in the side port.

\subsection{Automatic Indexing of EBSPs}

Several semi-automatic analysis procedures for the determination of crystallographic orientations from EBSPs are available (Dingley, Longden, Weinbren and Alderman, 1987, Schmidt, Bilde-Sørensen and Juul Jensen, 1991). Recently, work has been concentrated on developing image processing procedures by which the computer identifies elements (bands or poles) in EBSPs (Juul Jensen and Schmidt, 1990, Wright and Adams, 1991, Wright and Adams, 1992, Krieger Lassen, Juul Jensen and Conradsen, 1992). By combination of the two types of procedures, the analysis and image processing procedures, the indexing of EBSPs can be made fully automatic. 

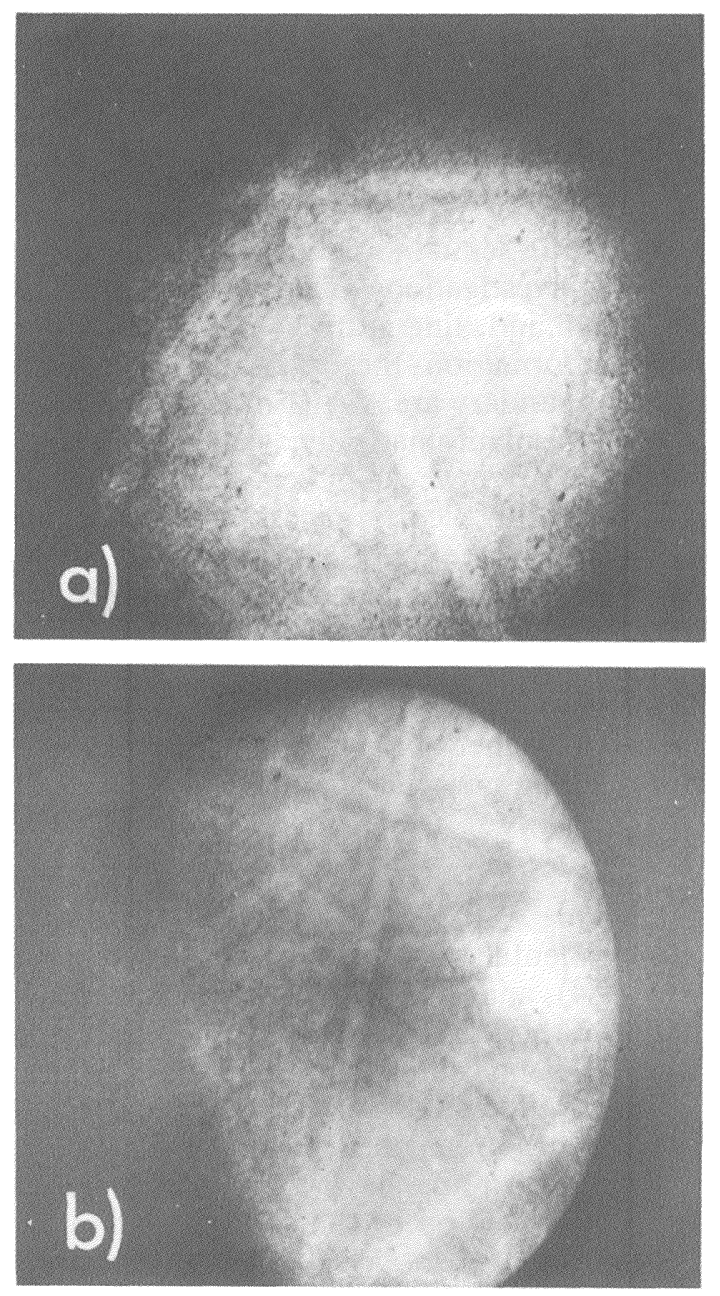

Figure 1 Typical EBSPs from aluminium a) Phosphor screen mounted in SEM rear port. b) Phosphor screen mounted in SEM side port.

Two such procedures are reviewed in the following:

1. Image processing: Computer identification of lines corresponding to bands in the EBSP (Krieger Lassen, Juul Jensen and Conradsen, 1992).

2. Analysis: Calculation of the crystallographic orientation based on the input of lines corresponding to bands in the EBSP (Schmidt, Bilde-Sørensen and Juul Jensen, 1991).

The two procedures have been shown to be very powerful in finding EBSP lines and indexing EBSPs, respectively. They are, however, not yet operating together. Indexing is thus not yet fully automatic, but it is discussed how this goal may be achieved. 


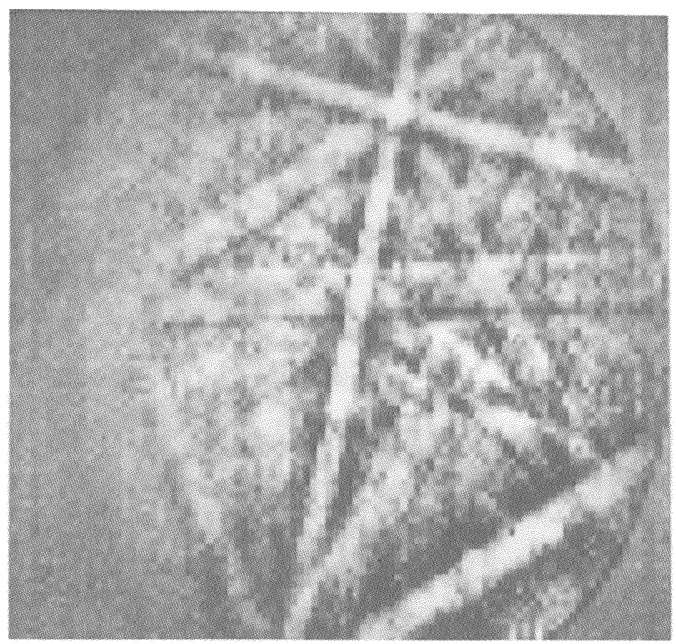

Figure 2 Reduced EBSP (corrected for background and reduced to $100 \times 100$ pixels) corresponding to Figure 1b.

Image processing The aim of this routine is to find lines corresponding to EBSP bands. First the original EBSP (see Figure 1b) is corrected for background and reduced to a $100 \times 100$ pixel picture by summation over $4 \times 4$ pixel squares in the original picture (only the central $400 \times 400$ parts of the original EBSP is considered). This reduction in the number of pixels significantly reduces the computer time needed later in the programme, and no important information is lost. The reduced picture corresponding to Figure $1 \mathrm{~b}$ is shown in Figure 2.

The position of the bands in the reduced image is found using a Hough transform. The standard Hough transform (Hough, 1962) is designed to find lines in binary images. Each point, $(x, y)$, in the picture different from zero is transformed to a sinusoidal curve

$$
R=x \cos \theta+y \sin \theta
$$

where $\theta$ is the range $0-180^{\circ}$ (see Figure 3 ). If the points $(x, y)$ lie on a straight line all the sinusoidal curves will intersect in a common point $(R, \theta)$ in the Hough space. The parameters $R, \theta$ describe the line (see Figure 3 ).

This simple procedure has to be somewhat modified to be optimal for finding lines in an EBSP picture (Figure 2). The EBSP picture is not binary, and too much information is lost in attempts to making it binary using various edge detection and thresholding techniques. Instead the EBSP picture is used as it is. Each pixel is transformed to the sinusoidal curve according to equation 2 with an intensity equal to the intensity of that pixel. This means, that an EBSP band will develop into a series of intense sinusoided curves which will intersect in a broad peak in the Hough space. The width of the peak depends on the width of the EBSP band. Figure 4 shows the result of this modified Hough transform.

Due to the nature of the Hough transform (Eq. 2) the peaks corresponding to lines of any width will be butterfly shaped (Van Veen and Groen, 1981, Leaves and Boyce, 1987). By using a butterfly filter the most intense points in the Hough 


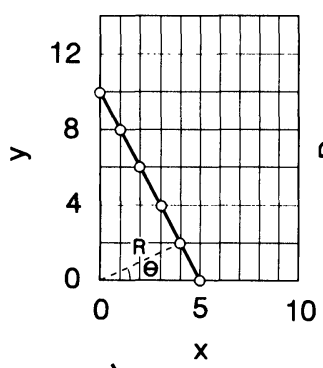

a)

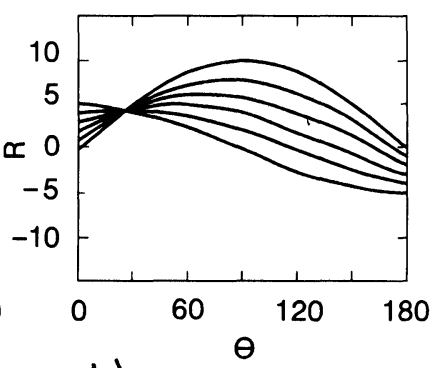

b)

Figure 3 Principles of the Hough transform. a) straight line in the $(x, y)$ image space. b) Hough curves of the $(x, y)$ points in a) shown in the $(R, \theta)$ Hough space.

space (Figure 4) are enhanced and identified. The parameters of the lines corresponding to center lines of EBSP bands are calculated from these Hough points using the fact that EBSP bands cross in common poles (Krieger Lassen et al., 1992). The result of this identification is shown in Figure 5. Here the computer identified lines are shown superimposed on the original EBSP. It can be seen that 15 lines have been identified. This very high number of correctly identified lines is typical. For a series of 25 EBSPs from commercially pure aluminium, from 12 to 16 , lines were identified in the different patterns. On average 14 lines were found.

Compared to other image processing procedures used for EBSP analysis (Juul Jensen and Schmidt, 1990, Wright and Adams, 1991, Wright and Adams, 1992), the present procedure seems rather effective. It requires no a priori information about the sample or microscope conditions, it is relatively simple to implement and it finds a significantly larger number of EBSP bands. For further information about the routine, see Krieger Lassen et al. (1992).

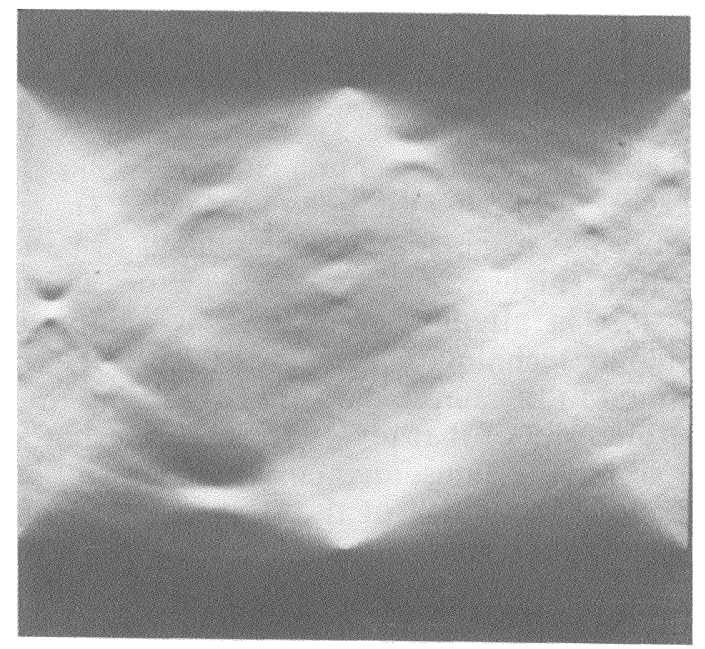

Figure 4 Peaks in the Hough space resulting from a modified Hough transform of Figure $1 \mathrm{~b}$. 


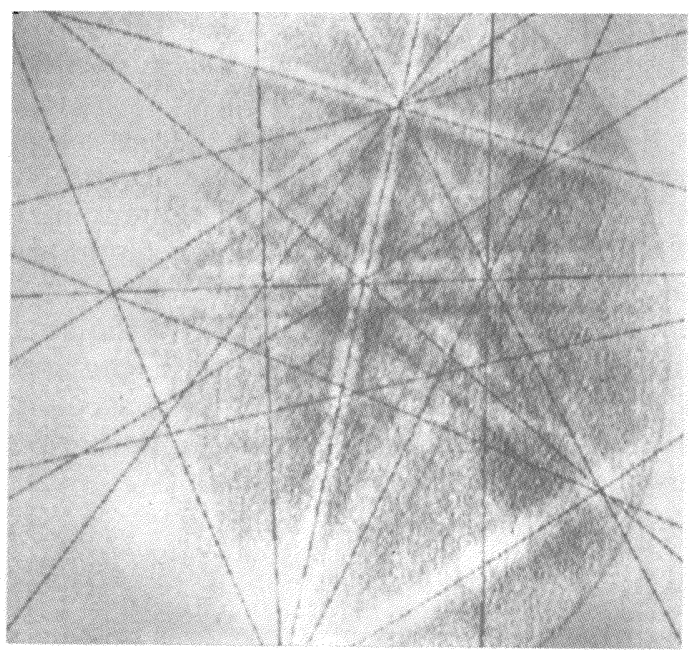

Figure 5 Lines identified by the image processing procedure (shown as dark lines) superimposed on the original EBSP (as in Figure 1b).

Analysis. The aim of this routine is to determine the crystallographic orientation from the input of lines corresponding to bands in the EBSP. Each EBSP band corresponds to crystal lattice plane $(h k l)$, and the determination of the crystallographic orientation of the EBSP is, in the present programme, entirely based on a comparison of interplanar angles.

Before any EBSPs from a given sample material can be analysed, a reference table containing the interplanar angles characteristic for that sample material has to be created. Each table is based on input information about the number of atoms per unit cell $(\mathrm{n})$, the atom positions $\left(x_{j}, y_{j}, z_{j}\right)$ and the atomic form factor $\left(f_{j}\right)$. The structure factor is calculated for all low index $(h k l)$ reflections.

$$
S(h k l)=\sum_{j=1}^{n} f_{j}(h k l) \exp \left(-i 2 \pi\left(h x_{j}-k y_{j}+l z_{j}\right)\right)
$$

The diffracted intensity of an $(h k l)$ EBSP band, is to a first approximation, proportional to $S(h k l) \times S^{*}(h k l)$ (Schmidt and Olesen, 1989).

Since only the strongest reflecting bands (bands with high values of $S(h k l) \times$ $S^{*}(h k l)$ ) are seen in an EBSP (typically less than 100), the interplanar angles between these are calculated (Young and Lytton, 1972) and stored in the reference table. For a given material it is only necessary to calculate the reference table once.

For each experimental EBSP, two or more lines are identified (at present by an operator). These are transformed to a set of three dimensional planes, and the corresponding interplanar angles are calculated. By comparison with the angles in the reference table the most likely solution is calculated and the corresponding simulated EBSP is superimposed on the experimental EBSP. The operator can accept or reject the solution. If rejected other possible solutions are tested. When three bands, not intersecting in a common pole are used as input, the correct solution is obtained at the first attempt in $80 \%$ of the cases.

For further information about the procedure, see Schmidt et al. (1991). 
For automated indexing, the next step is to use those lines identified by the Hough image processing procedure in the analysis procedure. Two approaches for doing this are considered:

One approach is to use three of the identified lines as input in the analysis calculation. Among the possible solutions, the correct one is selected by comparing the lines in the simulated EBSP to those found by the computer in the experimental EBSP. If all "experimental lines" correspond to one (and only one) of the simulated patterns, the solution is accepted. Another approach is directly to use all of the computer identified lines as input in the analysis calculation. In the latter case, the set of equations to calculate the orientation is largely overdetermined. A statistical estimation, taking into account the quality of the computer identified lines, is used to find a possible solution; only if this solution is unambigiously determined is the solution accepted.

\section{CONCLUDING REMARKS}

Microtexture techniques are powerful tools for studies of nucleation, growth of nuclei and the recrystallized structure. However, the time necessary to determine statistically reliable results is often a major problem. At present, work is underway to develop image processing procedures which automatically index EBSP patterns without the need for an operator to identify bands, poles etc. It is believed that during 1992 these routines will become as reliable as any of the semiautomatic procedures. Combined with computer controlled sample or beam movement, such routines will allow automatic orientation determinations. This will significantly improve the potential of the technique.

\section{ACKNOWLEDGMENTS}

N. Hansen, J. B. Bilde-Sørensen and N. C. Krieger Lassen are thanked for helpful discussions, and E. Sørensen for typing the manuscript.

\section{References}

Alam, M. N., Blackman, M. and Pashley, D. W. (1954). Proc. Royal Soc. A. 221, 224-242.

Bay, B. and Hansen, N. (1979). Met Trans. A. 10A, 279-288.

Bellier, S. P. and Doherty, R. D. (1977). Acta met. 25, 521-538.

Carr, M. J. (1982). JEOL News 20E, 35-37.

Chapman, P. F. and Stobbs, W. M. (1969). Phil. Mag. 19, 1015-1030.

Dadson, A. B. C. and Doherty, R. D. (1992). Acta metall. mater. 40, 345-352.

DeHoff, R. T. (1968). In Quantitative Microscopy. Eds. R. T. DeHoff and F. N. Rhines. McGraw-Hill NY 291-325.

Dingley, D. J., Longden, M., Weinbren, J. and Alderman, J. (1987). Scanning Microsc. 1, 451-456.

Dingley, D. J. (1981). Scanning Electron Microsc. 1981 IV, 273-286.

Dingley, D. J. (1984). Scanning Electron Microsc. 1984 II, 569-575.

Doherty, R. D. (1980). Recrystallization and Grain Growth of Multi-Phase and Particle Containing Materials. Eds. N. Hansen et al., Ris $\emptyset$ National Laboratory, Denmark 57-71.

Driver, J. (1992). In these proceedings.

English, A. T. and Backofen, W. A. (1964). Trans. AIME 230, 396-407.

Gottstein, G. (1988). ICOTOM 8. Eds J. S. Kallend and G. Gottstein. TMS. Warrendale 195-202. 
Hall, M. G. and Hutchinson, W. B. (1980). Metallurgist 12, 371-375.

Heimendahl, M., Bell, W. and Thomas, G. (1964). J. Appl. Phys. 35, 3614-3616.

Hirsch, P. B., Howie, A., Nicholson, R. B., Pashley, D. W. and Whelan, M. J. (1965). Electron Microscopy of Thin Crystals (Butterworth, London).

Hjelen, J. (1990). Thesis Universitetet Trondheim, $139 \mathrm{pp}$.

Hjelen, J., Ørsund, R. and Nes, E. (1991). Acta Metall. Mater. 39, 1377-1404.

Hough, P. V. C. (1962). U.S. Patent 3 069.654 Dec. 18, 1962.

Humphreys, F. J. (1983). Textures, Microstructures 6, 45-62.

Humphreys, F. J. (1988). ICOTOM 8. Eds. J. S. Kallend and G. Gottstein. TMS, Warrendale 171-182.

Humphreys, F. J. and Juul-Jensen, D. (1986). Annealing Processes-Recovery, Recrystallization and Grain Growth. Eds. N. Hansen et al., Ris $\varnothing$ National Laboratory, Denmark 93-106.

Juul Jensen, D., Hansen, N. and Humphreys, F. J. (1985). Acta metall. 33, 2155-2162.

Juul Jensen, D. and Randle, V. (1989). In Materials Architecture, Eds. J. B. Bilde-Sørensen et al., Ris $\varnothing$ National Laboratory $103-126$.

Juul Jensen, D. and Schmidt, N. H. (1990). Recrystallization '90. Eds. T. Chandra, TMS. Warrendale 219-224.

Klepeis, S. J., Benedict, J. P. and Anderson, R. M. (1988). Specimen Preparation for Transmission Electron Microscopy of Materials. Eds. Bravman et al., MRS, Pittsburgh, 179-184.

Krieger Lassen, N. C., Juul-Jensen, D. and Conradsen, K. (1992). Scanning Microscopy, 6, 115-121.

Leaves, V. F. and Boyce, J. F. (1987). Image and Vision Computing 5, 161-166.

Lindbo, J. (1991). Ris $\varnothing$ pıivate communication.

Newbury, D. E. and Yakowitz, H. (1975). In Practical Scanning Microscopy. Eds. Goldstein et al. (Plenum) 149-210.

Peters, E. T. and Ogilvie, R. E. (1965). Trans. met. Soc. AIME 233, 89-95.

Ralph, B. and Ecob, R. C. (1984). Microstructural Characterization of Materials by NonMicroscopical Techniques. Eds. N. Hessel Andersen et al., Ris $\emptyset$ National Laboratory, Denmark 109-129.

Randle, V. (1992). In these proceedings.

Schmidt, N. H., Bilde-Sørensen, J. B. and Juul Jensen, D. (1991). Scanning Microscopy, 5, 637-643.

Schmidt, N. H. and Olesen, N. Ø. (1989). Canadian Mineralogist 27, 15-22.

Schwarzer, R. and Weiland, H. (1984). ICOTOM 7. Eds. C. M. Brakman et al. Neth. Soc. Mat. Sci., Holland 839-843.

Van Veen, T. M. and Groen, F. C. A. (1981). Pattern Recognition 14, 137-145.

Venables, J. A., Harland, C. J. and bin-Jaya, R. (1976). Development in Electron Microscopy and Analysis. Academic Press, London, 101-104.

Watanabe, T. (1992). In these proceedings.

Weiland, H. and Schwarzer, R. A. (1984). ICOTOM 7. Eds. C. M. Brakman et al. Neth. Soc. Maths. Sci., Holland 857-862.

Weiland, H. (1991a). Proc. 49th Ann. Meet. Elec. Micros. Soc. Am. 792-793.

Weiland, H. (1991b). Alcoa Technical Center Report DE. 56-91DE-9 pp. 36.

Wright, S. I. and Adams, B. L. (1991). Metal. Trans. In press.

Wright, S. I. and Adams, B. L. (1992). In these proceedings.

Young, C. T. and Lytton, J. L. (1972). J. Appl. Phys. 43, 1408-1417. 